\title{
Shape-Persistent Actuators from Hydrazone Photoswitches
}

\author{
Alexander Ryabchun, ${ }^{\dagger, \S \odot ~ Q u a n ~ L i, ~}{ }^{\ddagger, \S}$ Federico Lancia, ${ }^{\dagger \odot}$ Ivan Aprahamian, ${ }^{*}, \ddagger \odot$ \\ and Nathalie Katsonis*, ${ }^{*}$ \\ ${ }^{\dagger}$ Bio-inspired and Smart Materials, MESA+ Institute for Nanotechnology, University of Twente, P.O. Box 207, 7500 AE Enschede, \\ The Netherlands \\ ${ }^{\ddagger}$ Department of Chemistry, Dartmouth College, 6128 Burke Laboratory, Hanover, New Hampshire 03755, United States
}

\section{Supporting Information}

ABSTRACT: Interfacing molecular photoswitches with liquid crystal polymers enables the amplification of their nanoscale motion into macroscopic shape transformations. Typically, the mechanism responsible for actuation involves light-induced molecular disorder. Here, we demonstrate that bistable hydrazones can drive (chiral) shape transformations in liquid crystal polymer networks, with photogenerated polymer shapes displaying a longterm stability that mirrors that of the switches. The mechanism involves a photoinduced buildup of tension in the polymer, with a negligible influence on the liquid crystalline order. Hydrazone-doped liquid crystal systems thus diversify the toolbox available to the field of lightadaptive molecular actuators and hold promise in terms of soft robotics.

S etting soft matter in motion with molecular machines will result in dynamic materials that can amplify chemical or light inputs into mechanical effects. However, harnessing the motion of artificial molecular motors and switches in producing useful work remains a daunting challenge ${ }^{1}$ that requires interfacing these dynamic molecules with soft matter. ${ }^{2}$ Polymeric systems have been used successfully in this context, ${ }^{3}$ and it is expected that the development of such materials into shape-shifting actuators that display a broad range of stable shapes will support innovation in soft robotics, photonics, microfluidics and biomedical applications. ${ }^{4,5}$ In this context, the action of molecular photoswitches has yielded large and complex shape transformations in liquid crystal (LC) polymer networks, where the anisotropy and collective behavior of LCs facilitate the emergence of macroscopic effects. ${ }^{6,7}$ Azobenzene photoswitches have played a major role in the advancement of research in this area; however, literature examples point at limitations in terms of kinetics, mechanical function, multistability and multiactuation modes. ${ }^{8}$ Azobenzene-based actuators also lack light-generated shape stability, because in most cases the $Z$ isomer is transient under dark. ${ }^{9}$ While we and others reported on LC polymers containing bistable fluorinated azobenzenes, ${ }^{10,11}$ the field calls for broadening the spectrum of photoswitches that can permanently lock the actuation of soft materials and, more generally, for lightresponsive switches with new energy landscapes, that can mediate the versatile behavior of moving polymers.
We recently reported on a novel family of negatively photochromic hydrazones, ${ }^{12,13}$ whose bistability motivated us to explore the implication of this effect on polymer actuation. Here we show how hydrazone H1 (Figure 1a) can be used to create a large range of stable shapes. Moreover, we show that upon photoisomerization, only a minor disruption of the LC order occurs, indicating that in the polymer counterpart, the origin of the light-induced shape transformation is not confined to light-induced order disruption, which is typically the major effect observed with azobenzenes. Instead, the major contribution originates from mechanical stresses that are generated by photoinduced molecular shape changes.

We designed hydrazone switches $\mathbf{H 1}$ and $\mathrm{H} 2$ bearing acrylate units in the rotor and/or stator part to facilitate their integration into cross-linked liquid crystalline polymers (Figure 1a). The rod-like shape and rigidity increase compatibility with the liquid crystals, so that they can be used as dopants with concentrations up to 5-6 wt \%. The switchable monomers were synthesized using a straightforward procedure (Scheme S1).

The photoisomerization of $\mathbf{H 1}$ was studied using ${ }^{1} \mathrm{H}$ NMR in toluene- $d_{8}$. Irradiating a sample of Z-H1 (>99\%) with $\lambda=$ $410 \mathrm{~nm}$ light yields a photostationary state (PSS) consisting of 95\% E-H2 (Figure S26), with a quantum yield of $\Phi_{E \rightarrow Z}=2.3 \pm$ $0.1 \%$ (Figure S30). Irradiating the resulting solution with $\lambda=$ $365 \mathrm{~nm}$ light yields a mixture consisting of $68 \%$ of the $Z$ isomer at PSS with $\Phi_{E \rightarrow Z}=9.1 \pm 0.4 \%$ (Figure S31). The compositions for different PSSs are gathered in Table S1. ${ }^{14}$ The isomerization was followed by UV/vis spectroscopy (Figure 1b), and no photodegradation was observed up to ten cycles (Figure S29). The determination of the thermal isomerization half-life of $E-\mathbf{H} \mathbf{1}$ in solution was hampered by the presence of the thermally reactive acrylate moiety. Therefore, we synthesized a model compound lacking the polymerizable groups and determined its half-life in DMSO to be $3435 \pm 50$ years at $298 \mathrm{~K}$ (Figures S32-38 and Table S2).

Before forming the liquid crystal polymer networks, we investigated how the switch aligns in the LC, to quantify the extent of dis/order stemming from each configuration. We doped a nematic LC with H1 (1 wt \%) and introduced the resulting liquid crystal into a planar cell. Linearly polarized light was used to characterize the orientation of both Z-H1 and E-H1 in the LC host (Figure 1c). The angular dependence of

Received: October 26, 2018

Published: January 9, 2019 
a)
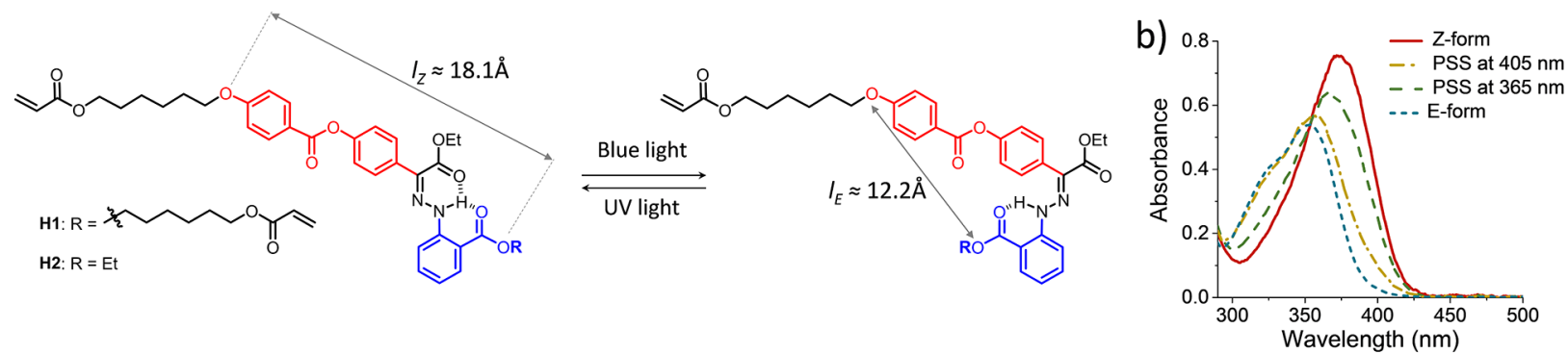

c)

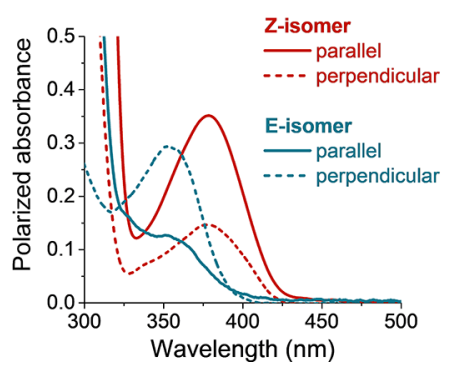

d)

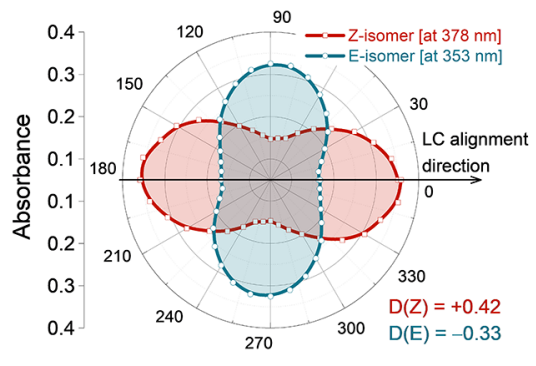

e)

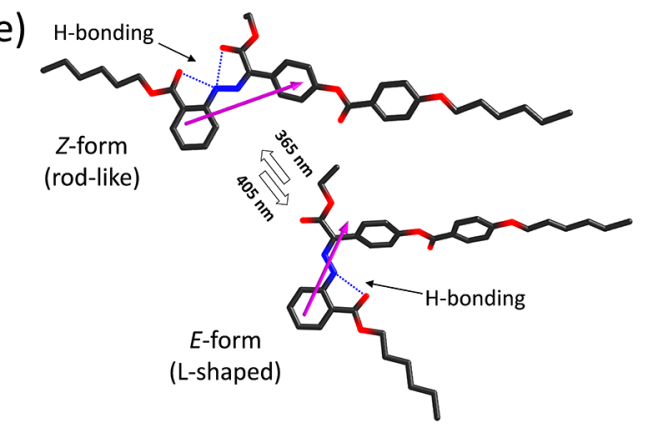

Figure 1. (a) Hydrazones $\mathbf{H 1}$ and $\mathbf{H 2}$. The stator and rotor are drawn in red and blue, respectively. $l_{Z}$ and $l_{E}$ are the calculated lengths of the switching moiety. (b) Absorbance spectra of $\mathbf{H 1}$ in toluene $\left(2.6 \times 10^{-5} \mathrm{M}\right) \cdot \varepsilon_{\max }(Z-\mathrm{H} \mathbf{1})=2.86 \times 10^{4} \mathrm{M}^{-1} \mathrm{~cm}^{-1}, \varepsilon_{\max }(E-\mathrm{H} \mathbf{1})=2.03 \times 10^{4} \mathrm{M}^{-1}$ $\mathrm{cm}^{-1}$. (c) Polarized absorbance spectra of $Z-\mathrm{H} 1$ and $E-\mathrm{H} 1$ in the unidirectionally aligned nematic host, and d) their angular dependence. Parallel and perpendicular directions are defined with respect to the LC alignment. The dichroism of the guest molecules is calculated as $D=\left(A_{\perp}-A_{\|}\right) /$ $\left(A_{\perp}+A_{\|}\right)$. (e) Calculated structures of Z-H1 and E-H1. The acrylic moieties are omitted for clarity. The purple arrows show the direction of the moment associated with the $S^{0}$ to $S^{1}$ electronic transition; their direction indicates how the switch interacts with polarized light.

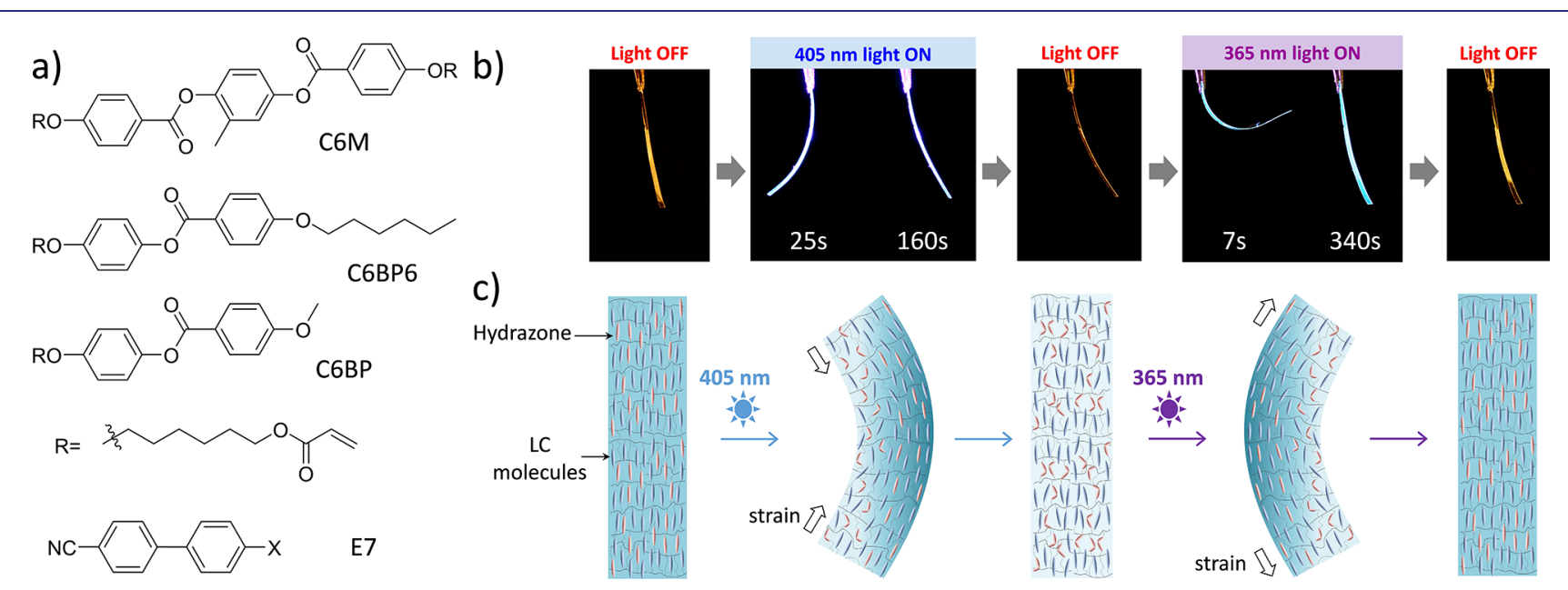

Figure 2. (a) Molecular components of the LC prepolymer. (b) Photoactuation of the polymer ribbons incorporating H1. Thickness $25 \mu \mathrm{m}$. (c) Representation of the photoinduced shape-shifting.

the polarized absorbance indicates that the rod-shaped Z-H1 aligns preferentially along the LC director (Figure 1d). In contrast, after photoisomerization, the polar plot indicates that the maximum polarized absorption of E-H1 becomes perpendicular to the LC director (Figure 1d). In Z-H1, the electronic transition moment associated with the $S^{0} \rightarrow S^{1}$ electronic transition is oriented along the stator, while in E-H1 it is localized along the rotor moiety (Figure 1e). Given the Lshape of E-H1, we conclude that even after switching, the mesogenic stator remains aligned along the LC director. Because only the rotor reorients its position in the liquid crystal, we conclude that the liquid crystal order is only minimally affected by photoconversion of the switch.

The absence of photoinduced disorder by hydrazone switching in liquid crystals is confirmed by the fact that the dichroism values of $\mathbf{H 1}$ measured before and after irradiation are similar (Figure 1d). Further, the dichroism values before and after photoisomerization are also similar for a well-known molecular alignment probe that is embedded in the liquid crystal (see Figure S40).

As polymerization preserves the alignment of liquid crystals, we conclude that, also in the polymer network, the photoisomerization does not yield any significant disorder. In liquid crystal networks, two effects can contribute to shape changes: (i) polymer tension as a result of changes in the shape of constituent molecules, and (ii) shrinkage/elongation of the network because of reduction of the order parameter. In the absence of light-induced disorder, we conclude that in the case of hydrazones, any shape-shifting event should be rooted in the molecular shape changes of the switch primarily. 
Next, we incorporated the switches covalently in a photopolymerizable LC (Figure $2 \mathrm{a})^{15,16}$ After photopolymerization, polymer ribbons were cut along the molecular alignment. When Z-H2 was incorporated as a side chain, no shape changes were ever observed under illumination (Figure S41). In contrast, when the switch was incorporated as a crosslinker, large shape transformations were observed under illumination (Figure 2b). This observation points at a shapeshifting mechanism based on photogenerated stresses, because only cross-linked molecular switches induce such a mechanical action, in contrary to pendant side-chains. ${ }^{17}$ Also considering that the liquid crystalline order is not disrupted by photoisomerization, we conclude that, in hydrazone LC networks, the mechanical stresses are imposed by the action of the switches on the polymer network. With this mechanism, the shortening of the cross-linking length upon isomerization and subsequent contraction of the network likely become key (the difference in lengths is $\approx 0.6 \mathrm{~nm}$ ).

In the initial stages of illumination $(\lambda=405 \mathrm{~nm})$, a polymer ribbon incorporating Z-H1 bends toward the light, and as irradiation proceeds, the ribbon bends back away. Switching off the light does not result in further shape changes, which confirms that the shape transformation is not related to any light-induced temperature increase. Overall, we propose that the back-and-forth bending originates from the isomerization of the switches that are located next to the irradiated surface, whereas the switches on the other side of the sample do not isomerize. Such a gradient photogenerates mechanical stress (Figure 2c). With further irradiation, and thanks to the negative photochromicity of the switch (i.e., going from colored to transparent upon isomerization), isomerization develops throughout the entire thickness of the sample until reaching PSS, and the ribbon bends back to its original shape. At PSS, the ribbon displays a minor curvature in comparison with the original flat shape: a fact that we attribute to a small gradient in the cross-linking density, which results from photopolymerization. Subsequent irradiation with UV light $(\lambda=365 \mathrm{~nm})$ results in a sharp gradient in $Z$-isomer concentration, because of the efficient absorption of the $E$ isomer, and thus in the rapid bending away from the light source. Further exposure to UV light completes the conversion into the $Z$-isomer and causes unbending (Figure $3 a$ ).

We note that increasing the light intensity accelerates the actuation process (Figure S42): with high intensities, a higher curvature is reached, likely as a result of heating, that reduces the order of the network.

The stability of both the $Z$ - and E-forms allows fixing any isomeric composition, simply by adjusting the total illumination time, resulting in photogenerated shapes that are stable for months (Figure 3b, S43). Moreover, hydrazone-activated polymers display large shape transformations and versatility in their actuation modes, e.g., by making use of splay organization (Figure S44, S45).

In LC polymer networks, gradients in molecular orientation determine the shape of the polymer and, to a certain extent, also its shape-transformation properties. The latter can also be designed by gradients in cross-linking density, by patterning, or using multilayers. ${ }^{18-21}$ We sought to take advantage of the bistability of hydrazones and of their strong absorbance in both forms, to photogenerate strong gradients of hydrazone isomer concentrations, across the polymer film. As the switches change in length upon photoswitching, the photogenerated
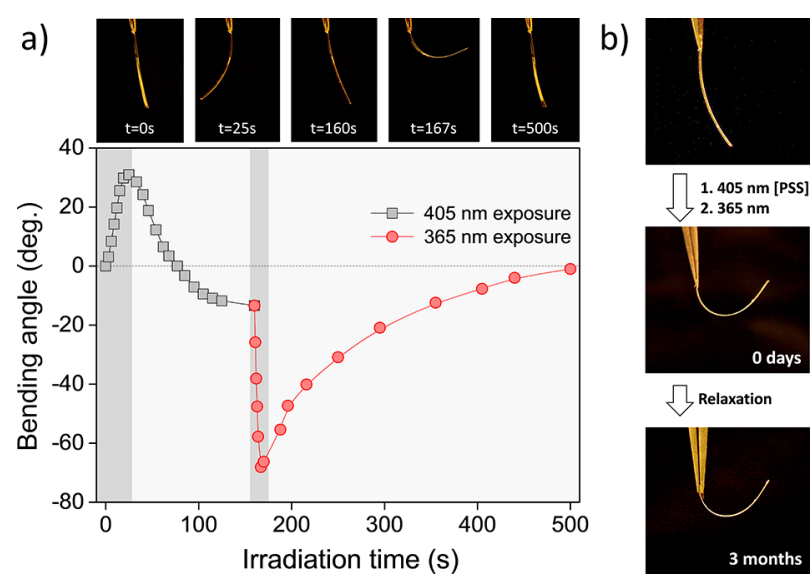

Figure 3. (a) Bending angle of the ribbon as a response to illumination. The molecules are aligned along the long axis of the ribbon. The dark gray areas correspond to stages where photogenerated stress builds up. (b) Stability of the curvature mirrors that of the photogenerated gradient.

gradients in concentration translate into strong gradients of mechanical strain, and thus into robust and chiral deformation.

We illuminated a hydrazone-containing polymer (Mixture 2, Table S3) using a two-step procedure. A flat ribbon doped with Z-H1 (Figure 4a) was first irradiated with visible light to
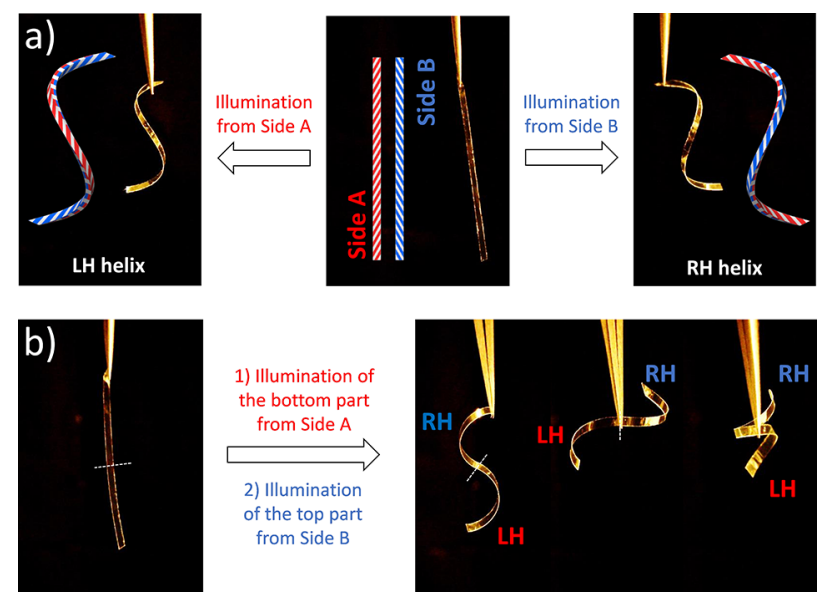

Figure 4. Macroscopic chirality resulting from the introduction of gradients of mechanical strain. The white stripes indicate the molecular orientation in the sample. Opposite sides of the ribbon are colored in red and blue as a guide for the eyes. ( $a, b)$ Flat ribbon with molecular alignment tilted $\sim 22^{\circ}$ with respect to the long axis. The stepwise illumination this flat ribbon includes exposure to blue light, then to UV light. Side A was irradiated during photopolymerization and is thus characterized by a higher cross-linking density. $\mathrm{LH}$ and $\mathrm{RH}$ indicate left- and right-handedness, respectively.

convert the switches into $E-\mathbf{H} \mathbf{1}$, followed by irradiation with UV light. Because $\varepsilon_{365}(E-\mathbf{H} \mathbf{1})>\varepsilon_{405}(Z-\mathbf{H} \mathbf{1})$, this two-step procedure creates a sharp gradient in the concentration of $Z$ H1. Overall, the process leads to a gradient of mechanical strain, which eventually translates into an out-of-plane chiral deformation, the handedness of which is determined by which side of the ribbon is illuminated (Figure $4 a$ ). ${ }^{22}$ A variety of shapes can be prepared (Figure 4b), simply by cutting ribbons at different angles with respect to the direction of molecular alignment (Figure S46), and the steeper the gradient of mechanical strain, the higher the curvature of the ribbons 
(Figure S47). All these photogenerated shapes demonstrate a long-term stability that mirrors that of the switches.

In conclusion, a hydrazone photoswitch was integrated into a liquid crystal to yield a polymer network that responds to light with large shape transformations. The original photochemistry of the switch supports multistability, persistence of the photogenerated shapes, versatility in shape-shifting modes, and the formation of chiral materials from otherwise achiral molecules, thus starting to address long-standing issues in the field. ${ }^{4}$ From a mechanistic point of view, we show that the design of molecular switches has a determining influence on the mechanism of photoactuation, and should thus allow for the engineering of adaptive materials with new distinct features. Overall, this photochemical system expands the toolbox available to set soft matter in motion and adds to the capabilities of polymer photoactuation.

\section{ASSOCIATED CONTENT}

\section{S Supporting Information}

The Supporting Information is available free of charge on the ACS Publications website at DOI: 10.1021 /jacs.8b11558.

General methods, synthesis and characterization of switches, photoisomerization studies, quantum yields and kinetic studies, polymer characterization (PDF)

\section{AUTHOR INFORMATION}

\section{Corresponding Authors}

*n.h.katsonis@utwente.nl

*ivan.aprahamian@dartmouth.edu

\section{ORCID}

Alexander Ryabchun: 0000-0001-9605-3067

Federico Lancia: 0000-0003-3075-1465

Ivan Aprahamian: 0000-0003-2399-8208

Nathalie Katsonis: 0000-0003-1054-6544

\section{Author Contributions}

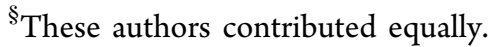

\section{Notes}

The authors declare no competing financial interest.

\section{ACKNOWLEDGMENTS}

We acknowledge financial support from the Volkswagen Foundation and the Dutch Science Foundation NWO (Projectruimte Grant 13PR3105). We thank Dr. D. Morozov (University of Jyväskylä, Finland) for calculations of molecular geometry and electron transitions moments, and Dr. I. Raguzin (Leibniz Institute of Polymer Research, Dresden, Germany) for assistance with illustrations.

\section{REFERENCES}

(1) (a) Browne, W. R.; Feringa, B. L. Making Molecular Machines Work. Nat. Nanotechnol. 2006, 1, 25-35. (b) Coskun, A.; Banaszak, M.; Astumian, R. D.; Stoddart, J. F.; Grzybowski, B. A. Great Expectations: Can Artificial Molecular Machines Deliver on their Promise? Chem. Soc. Rev. 2012, 41, 19-31. (c) Zhang, L.; Marcos, V.; Leigh, D. A. Molecular Machines with Bio-inspired Mechanisms. Proc. Natl. Acad. Sci. U. S. A. 2018, 115, 9397-9404. (d) Abendroth, J. M.; Bushuyev, O. S.; Weiss, P. S.; Barrett, C. J. Controlling Motion at the Nanoscale: Rise of the Molecular Machines. ACS Nano 2015, 9, 7746-7768. (e) Ornes, S. What's the best way to build a molecular machine? Proc. Natl. Acad. Sci. U. S. A. 2018, 115, 9327-9330.

(2) (a) Eelkema, R.; Pollard, M. M.; Vicario, J.; Katsonis, N.; Ramon, B. S.; Bastiaansen, C. W.; Broer, D. J.; Feringa, B. L.
Molecular Machines: Nanomotor Rotates Microscale Objects. Nature 2006, 440, 163. (b) Chen, J.; Leung, F. K-C.; Stuart, M. C. A.; Kajitani, T.; Fukushima, T.; van der Giessen, E.; Feringa, B. L. Artificial Muscle-Like Function From Hierarchical Supramolecular Assembly of Photoresponsive Molecular Motors. Nat. Chem. 2017, 10, 132-138.

(3) (a) Klajn, R.; Bléger, D. Integrating Macromolecules with Molecular Switches. Macromol. Rapid Commun. 2018, 39, 1700827. (b) Fredy, J. W.; Méndez-Ardoy, A.; Kwangmettatam, S.; Bochicchio, D.; Matt, B.; Stuart, M. C. A.; Huskens, J.; Katsonis, N.; Pavan, G. M.; Kudernac, T. Molecular Photoswitches Mediating the Strain-Driven Disassembly of Supramolecular Tubules. Proc. Natl. Acad. Sci. U. S. A. 2017, 114, 11850-11855.

(4) (a) Morone, M. I. Harnessing the Power of Shape-Shifting Polymers. Chem. Eng. News 2018, 96, 36. (b) Davenport, M. Packing More Punch Into Polymer Devices. Chem. Eng. News 2017, 95, 11.

(5) Fischer, P.; Palagi, S. Bioinspired Microrobots. Nat. Rev. Mater. 2018, 3, 113-124.

(6) White, T. J.; Broer, D. J. Programmable and Adaptive Mechanics with Liquid Crystal Polymer Networks and Elastomers. Nat. Mater. 2015, 14, 1087-1098.

(7) (a) Iamsaard, S.; Asshoff, S. J.; Matt, B.; Kudernac, T.; Cornelissen, J. J. L. M.; Fletcher, S. P.; Katsonis, N. Conversion of Light Into Macroscopic Helical Motion. Nat. Chem. 2014, 6, 229235. (b) A $\beta$ hoff, S. J.; Sukas, S.; Yamaguchi, T.; Hommersom, C. A.; Le Gac, S.; Katsonis, N. Superstructures of Chiral Nematic Microspheres as All-Optical Switchable Distributors of Light. Sci. Rep. 2015, 5, 14183. (c) Orlova, T.; Aßhoff, S. J.; Yamaguchi, T.; Katsonis, N.; Brasselet, E. Nat. Commun. 2015, 6, 7603. (d) Gelebart, A. H.; Mulder, D. J.; Varga, M.; Konya, A.; Vantomme, G.; Meijer, E. W.; Selinger, R.; Broer, D. J. Making Waves in a Photoactive Polymer Film. Nature 2017, 546, 632-635. (e) Lahikainen, M.; Zeng, H.; Priimagi, A. Reconfigurable Photoactuator Through Synergistic Use of Photochemical and Photothermal Effects. Nat. Commun. 2018, 9, 4148. (f) Ryabchun, A.; Lancia, F.; Nguindjel, A. D.; Katsonis, N. Humidity-Responsive Actuators From Integrating Liquid Crystal Networks in an Orienting Scaffold. Soft Matter 2017, 13, 8070-8075.

(8) Harris, J. D.; Moran, M. J.; Aprahamian, I. New Molecular Switch Architectures. Proc. Natl. Acad. Sci. U. S. A. 2018, 115, 94149422.

(9) For exception, see: Knie, C.; Utecht, M.; Zhao, F.; Kulla, H.; Kovalenko, S.; Brouwer, A. M.; Saalfrank, P.; Hecht, S.; Bléger, D. ortho-Fluoroazobenzenes: Visible Light Switches with Very LongLived Z Isomers. Chem. - Eur. J. 2014, 20, 16492-16501.

(10) (a) Iamsaard, S.; Anger, E.; Aßhoff, S. J.; Depauw, A.; Fletcher, S. P.; Katsonis, N. Fluorinated Azobenzenes for Shape-Persistent Liquid Crystal Polymer Networks. Angew. Chem., Int. Ed. 2016, 55, 9908. (b) Hendrikx, M.; ter Schiphorst, J.; van Heeswijk, E. P.; Koçer, G.; Knie, C.; Bléger, D.; Hecht, S.; Jonkheijm, P.; Broer, D. J.; Schenning, A. P. Re- and Preconfigurable Multistable Visible Light Responsive Surface Topographies. Small 2018, 14, 1803274.

(11) Kumar, K.; Knie, C.; Bléger, D.; Peletier, M. A.; Friedrich, H.; Hecht, S.; Broer, D. J.; Debije, M. G.; Schenning, A. P. H. J. A Chaotic Self-Oscillating Sunlight-Driven Polymer Actuator. Nat. Commun. 2016, 7, 11975 .

(12) (a) Qian, H.; Pramanik, S.; Aprahamian, I. Photochromic Hydrazone Switches with Extremely Long Thermal Half-Lives. J. Am. Chem. Soc. 2017, 139, 9140-9143. (b) Li, Q.; Qian, H.; Shao, B.; Hughes, R. P.; Aprahamian, I. Building Strain with Large Macrocycles and Using It To Tune the Thermal Half-Lives of Hydrazone Photochromes. J. Am. Chem. Soc. 2018, 140, 11829-11835. (c) Shao, B.; Baroncini, M.; Qian, H.; Bussotti, L.; Di Donato, M.; Credi, A.; Aprahamian, I. Solution and Solid-State Emission Toggling of a Photochromic Hydrazone. J. Am. Chem. Soc. 2018, 140, 1232312327. (d) Moran, M. J.; Magrini, M.; Walba, D. M.; Aprahamian, I. Driving a Liquid Crystal Phase Transition Using a Photochromic Hydrazone. J. Am. Chem. Soc. 2018, 140, 13623-13627.

(13) (a) Su, X.; Lessing, T.; Aprahamian, I. The Importance of the Rotor in Hydrazone-Based Molecular Switches. Beilstein J. Org. Chem. 
2012, 8, 872-876. (b) Ray, D.; Foy, J. T.; Hughes, R. P.; Aprahamian, I. A Switching Cascade of Hydrazone-Based Rotary Switches Through Coordination-Coupled Proton Relays. Nat. Chem. 2012, 4, 757-762.

(c) Foy, J. T.; Ray, D.; Aprahamian, I. Regulating Signal Enhancement with Coordination-Coupled Deprotonation of a Hydrazone Switch. Chem. Sci. 2015, 6, 209-213. (d) Tatum, L.; Foy, J. T.; Aprahamian, I. Waste Management of Chemically Activated Switches: Using a Photoacid to Eliminate Accumulation of Side Products. J. Am. Chem. Soc. 2014, 136, 17438-17441. (e) Qian, H.; Aprahamian, I. An Emissive and $\mathrm{pH}$ Switchable Hydrazone-Based Hydrogel. Chem. Commun. 2015, 51, 11158-11161. (f) Pramanik, S.; Aprahamian, I. Hydrazone Switch-Based Negative Feedback Loop. J. Am. Chem. Soc. 2016, 138, 15142-15145. (g) Aprahamian, I. Hydrazone Switches and Things in Between. Chem. Commun. 2017, 53, 6674-6684.

(14) The quantum yield and PSS measurements in solution were performed with a different light source $(\lambda=410 \mathrm{~nm})$.

(15) Iamsaard, S.; Villemin, E.; Lancia, F.; A $\beta$ hoff, S. J.; Fletcher, S. P.; Katsonis, N. Preparation of Biomimetic Photoresponsive Polymer Springs. Nat. Protoc. 2016, 11, 1788.

(16) The compositions of the monomeric mixtures are provided in Table S3. Mixture 1 was used unless otherwise mentioned.

(17) Light-induced actuation by photothermal effect was observed in polymers incorporating a hydrazone as a side chain; see: Vantomme, G.; Gelebart, A. H.; Broer, D. J.; Meijer, E. W. A Four-Blade LightDriven Plastic Mill Based on Hydrazone Liquid-Crystal Networks. Tetrahedron 2017, 73, 4963-4967.

(18) Jeon, S. J.; Hauser, A. W.; Hayward, R. C. Shape-Morphing Materials from Stimuli-Responsive Hydrogel Hybrids. Acc. Chem. Res. 2017, 50, 161-169.

(19) Thérien-Aubin, H.; Wu, Z. L.; Nie, Z.; Kumacheva, E. Multiple Shape Transformations of Composite Hydrogel Sheets. J. Am. Chem. Soc. 2013, 135, 4834-4839.

(20) Wu, Z. L.; Moshe, M.; Greener, J.; Therien-Aubin, H.; Nie, Z.; Sharon, E.; Kumacheva, E. Three-dimensional Shape Transformations of Hydrogel Sheets Induced by Small-Scale Modulation of Internal Stresses. Nat. Commun. 2013, 4, 1586.

(21) Deng, J.; Li, J.; Chen, P.; Fang, X.; Sun, X.; Jiang, Y.; Weng, W.; Wang, B.; Peng, H. Tunable Photothermal Actuators Based on a PreProgrammed Aligned Nanostructure. J. Am. Chem. Soc. 2016, 138, $225-230$.

(22) Warner, M.; Terentjev, E. M. Liquid crystal elastomers; Oxford University Press: Oxford, UK, 2003. 